\title{
Stimulated Brillouin Scattering in High-Power Photonic Crystal Fiber Lasers in Different Pump Schemes
}

\author{
Abouricha $\mathbf{M}^{1 *}$, Boulezhar $\mathrm{A}^{1}$, Amrane $^{2}$ and Azami $\mathbf{N}^{2}$ \\ ${ }^{1}$ Laboratory of Renouwable Energy and System, Dynamics Faculty of Sciences Ain Chock, Hassan II University Casablanca, Morocco \\ ${ }^{2}$ National Institute of Posts and Telecommunications, Rabat, Morocco
}

\begin{abstract}
We present in this paper the special structure of photonic crystal fiber (PCF), the temperature-dependent $\mathrm{Yb}^{3+}$ photonic crystal fiber lasers model with stimulated Brillouin scattering (SBS) is presented by solving the steady-state rate equations with the (SBS) in the linear cavity. The numerical results show that the pump power, laser power and stokes powers propagating along axial positions are obtained by using the finite difference method and shooting method. The comparison results of the photonic crystal fiber laser model without temperature factor, the output powers and the SBS threshold powers in different pump schemes are obtained in the simulation paragraph. The numerical results show that the SBS threshold power in the two-end pump scheme is more noticeable than other pumps schemes.
\end{abstract}

Keywords: Stimulated Brillouin scattering; PCF; Fiber optic; Pump schemes

\section{Introduction}

$\mathrm{Yb}^{3+}$-doped photonic crystal fiber lasers pumped by laser diodes have more attracted attention in recent years, in several applications such as view commercial and military applications thanks to excellent beam quality, their high brightness, efficient heat dissipation, eminent efficiency, good compactness, etc., by comparison with traditional lasers such assolid-state or gas $[1,2]$. With the vailability of high-power laser diode bars and clad-pumping techniques, the output power of YDDC fiber lasers is able to reach hundreds watts, even 1000 watt, in the regime of the continuous-waves (CW) [3-5]. But, the Extensibility of output powers can be limited by nonlinear processes and amplified spontaneous emission such as stimulated Raman scattering, the optical Kerr effect and stimulated Brillouin scattering (SBS). Although these nonlinear effects could be of interest for specific applications [6-9]. The maximum SBS threshold pump power is theoretically obtained by achieving high power output scalability and narrowing the line-width of the fiber laser, and 70\% optical-optical efficiency was experimentally observed with $310 \mathrm{~W}$ total pump power at $976 \mathrm{~nm}$ [10]. Due to the presence of the first-order Stokes waves initiated by forward and backward pump power, the output laser power increases slower with the increase of pump power under bidirectional end-pumping [11]. A numerical analysis of SBS in high power linear cavity $\mathrm{Yb}^{3+}$-doped double-clad fiber lasers is investigated, the SBS threshold power can be improved significantly by broadening laser line-width, effectively by using large mode area fiber, shortening cavity length and reducing input mirror reflectivity at Stokes wavelength [12]. In addition, the temperature factor has practically no effect on the corresponding laser output power to SBS threshold power[13], they can also lead to some unexpected in stabilities in the laser signal. In particular, the SBS is expected to be the origin of instabilities in high-power fiber lasers [9] or deformation of pulses in fiber amplifiers [14]. The aim of this paper is to investigate theoretically the dependence of the SBS on system parameters in YDDC fiber lasers and the pumps schemes. By solving a set of laser rate equations with the SBS, the SBS thresholds are obtained under different fiber conditions. The results and analysis is presented facilitate the design and optimization of $\mathrm{Yb}^{3+}$-doped photonic crystal fiber lasers.

\section{Theoretical Model}

\section{Pump schemes}

The high power linear cavity $\mathrm{Yb}^{3+}$-doped fiber laser with SBS is described schematically in Figure 1 . The rate rate equations with temperature factor in the steady-state [15] and SBS in high power $\mathrm{Yb}^{3+}$-doped fiber laser are described by the nonlinear coupled rate Equations. (1)-(4). In our numerical model, $\mathrm{Yb}^{3+}$-doped fiber laser, signal stimulated emission and absorption, stimulated emission at the pump wavelength and scattering losses; both for the pump and signal the are considered; but excited state absorption (ESA) and spontaneous emission are negligible, for high pumping conditions [16,17].

\section{Rate equations with the SBS}

$$
\frac{N_{2}(z)}{N}=\frac{\frac{\Gamma_{p} \lambda_{p} \sigma_{a p} f_{l p}}{h c A}\left[P_{p}^{-}(z)+P_{p}^{+}(z)\right]+\frac{\Gamma_{s} \lambda_{s} \sigma_{a s} f_{l s}}{h c A}\left[P_{s}^{-}(z)+P_{s}^{+}(z)\right]}{\frac{\Gamma_{p} \lambda_{p}\left(\sigma_{a p} f_{l p}+\sigma_{e p} f_{u p}\right)}{h c A}\left[P_{p}^{-}(z)+P_{p}^{+}(z)\right]+\frac{\Gamma_{s} \lambda_{s}\left(\sigma_{a s} f_{l s}+\sigma_{e s} f_{u s}\right)}{h c A}\left[P_{s}^{-}(z)+P_{s}^{+}(z)\right]+\frac{f_{u s}}{\tau}}
$$

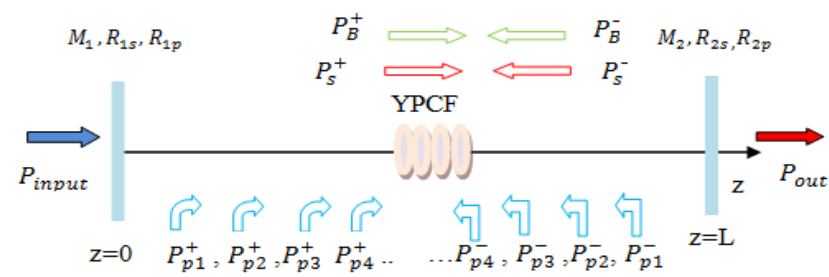

Figure 1: Schematic illustration of high power $\mathrm{Yb}^{3+}$-doped photonic crystal fiber laser with different pump schemes.

*Corresponding author: Abouricha M, Laboratory of Renouwable Energy and System, Dynamics Faculty of Sciences Ain chock, Hassan II University Casablanca, Morocco, Tel: 0614000 400; E-mail: mmabouricha@gmail.com

Received November 22, 2017; Accepted December 04, 2017; Published December 20, 2017

Citation: Abouricha M, Boulezhar A, Amrane S, Azami N (2017) Stimulated Brillouin Scattering in High-Power Photonic Crystal Fiber Lasers in Different Pump Schemes. J Laser Opt Photonics 4: 170. doi: 10.4172/2469-410X.1000170

Copyright: (c) 2017 Abouricha M, et al. This is an open-access article distributed under the terms of the Creative Commons Attribution License, which permits unrestricted use, distribution, and reproduction in any medium, provided the original author and source are credited. 


$$
\begin{aligned}
& \pm \frac{d P_{s}^{ \pm}(z)}{d z}=\Gamma_{s}\left[\left(\sigma_{a s} f_{l s}+\sigma_{e s} f_{u s}\right) N_{2}(z)-\sigma_{a s} f_{l s} N\right] P_{s}^{ \pm}(z)-\alpha_{s} P_{s}^{ \pm}(z)-\frac{g_{B}}{A_{e f f}} P_{B}^{ \pm}(z) P_{s}^{\mp}(z) \\
& \pm \frac{d P_{p}^{ \pm}(z)}{d z}=\Gamma_{p}\left[\left(\sigma_{a p} f_{l p}+\sigma_{e p} f_{u p}\right) N_{2}(z)-\sigma_{a p} f_{l p} N\right] P_{p}^{ \pm}(z)-\alpha_{p} P_{p}^{ \pm}(z) \\
& \pm \frac{d P_{B}^{ \pm}(z)}{d z}=-\alpha_{B} P_{B}^{ \pm}(z)+\frac{g_{B}}{A_{e f f}} P_{B}^{ \pm}(z) P_{s}^{\mp}(z) \\
& \text { Where: }
\end{aligned}
$$

$\mathrm{N}_{2}(\mathrm{z})$ is the upper level population density, $\mathrm{N}$ is the total doping population density of $\mathrm{Yb}$ ions.

$P_{p}^{ \pm}(z), P_{S}^{ \pm}(z)$ and $P_{B}^{ \pm}(z)$ are the signal power, laser pump power and first-order Brillouin Stokes power along the fiber, respectively. $\lambda_{s}$ and $\lambda_{p}$ are the laser signal and pump wavelengths, respectively.

The minus - and plus + superscripts represent propagation along the negative or positive $\mathrm{z}$-direction, respectively. $\Gamma_{p}$ and $\Gamma_{s}$ are the pump filling factor and laser signal filling factor in the fiber core, respectively. The expressions of these factors are the following:

$$
\begin{aligned}
& \Gamma_{s}=1-\exp \exp \left(-\frac{2 a^{2}}{\omega^{2}}\right) \\
& \Gamma_{p}=a^{2} / b^{2}
\end{aligned}
$$

Where $\omega$ is the field radius, $\mathrm{b}$ and $a$ are the radius of the fiber core and inner cladding.

$$
\frac{\omega}{a} \approx 0.65+1.619 \mathrm{~V}^{-\frac{3}{2}}+2.879 \mathrm{~V}^{-6} \quad[18,19] . \quad \sigma_{e s} \text { and } \sigma_{a s} \text { are the }
$$
emission cross-section and laser signal absorption, respectively. $\sigma_{e p}$ and $\sigma_{a p}$ are the emission cross-section and pump absorption, respectively. The scattering losses for the laser signal and pump powers are given by $\alpha_{s}$ and $\alpha_{p}$, respectively. The value of (SBS) is less than 0.1 $\mathrm{nm}$ away from the laser wavelength therefore, $\alpha=\alpha_{S} \mathrm{~h}, \mathrm{c}, \tau$ and $\mathrm{A}$ are the Planck's constant, light velocity spontaneous lifetime and $\mathrm{Yb}^{3+}$-doped area, respectively. $g_{B}$ is the SBS gain, $g_{B}=g_{0} \Delta v_{B} /\left(\Delta v_{B}+\Delta v_{S}\right)$, where $\mathrm{g}_{0}, \Delta v_{B}$ and $\Delta v_{s}$ are the intrinsic SBS gain constant, SBS gain bandwidth and laser bandwidth [18]. The effective core area $\mathrm{A}_{\text {eff }}$ is defined as $\pi r^{2} . f_{l s}\left(f_{l p}\right)$ and $f_{u s}\left(f_{u p}\right)$ are the Boltzmann occupation factor within upper and lower manifolds for the upper and lower levels of the laser (pump) transition $[16,20]$. At the pump wavelength $\lambda_{\mathrm{p}}=975$ $n m$ and signal wavelength $\lambda_{s}=1080 \mathrm{~nm}, f_{l s}\left(f_{l p}\right)$ and $f_{u s}\left(f_{u p}\right)$ are shown in Figure 2 and defined as follows:

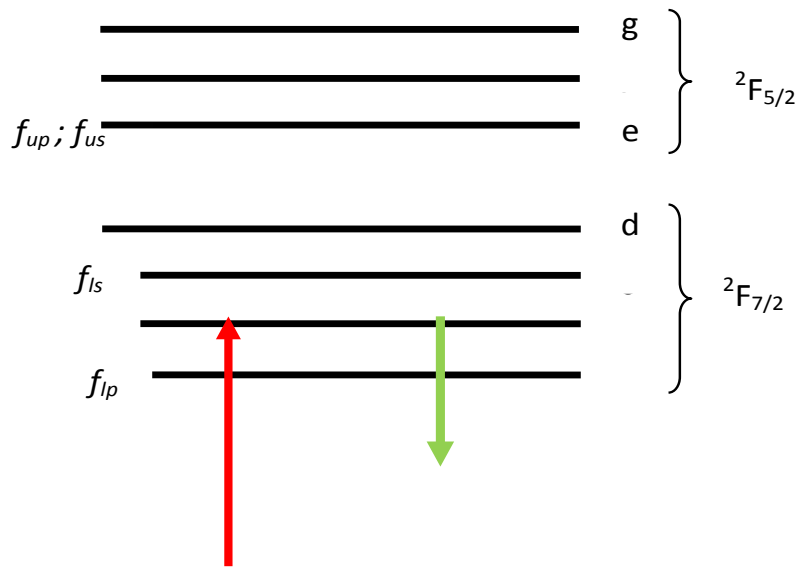

Figure 2: The energy level diagram showing the pump and laser transitions.
- Yb excited state:

$$
f_{u s}=f_{u p}=\frac{\exp \left(-\frac{E_{e}}{k T}\right)}{\sum_{x=a}^{g} \exp \left(-\frac{E_{x}}{k T}\right)}
$$

- $\mathrm{Yb}$ ground state:

$$
f_{l p}=\frac{\exp \left(-\frac{E_{a}}{k T}\right)}{\sum_{x=a}^{d} \exp \left(-\frac{E_{x}}{k T}\right)}
$$

- and:

$$
f_{l s}=\frac{\exp \left(-\frac{E_{c}}{k T}\right)}{\sum_{x=a}^{d} \exp \left(-\frac{E_{x}}{k T}\right)}
$$

Where:

$\mathrm{E}_{x}$ is the energy level difference between levels $x$ and $a$ in the $\mathrm{Yb}$ ground state and excited state, as shown in Table 1 [21,22].

$\mathrm{k}$ is the Boltzmann constant; $\mathrm{T}$ is the temperature distribution in the fiber core area, expressed by the following [23]:

$$
T(r)=T_{0}-\frac{Q(z) \cdot r^{2}}{\pi k_{1}} \text { if }(0 \leq r \leq a)
$$

Where $\mathrm{T}_{0}$ represents the temperature of fiber axis $(r=0), \mathrm{k}_{1}$ is the thermal conductivity of material and $T_{c}$ is the environment temperature, such as $\mathrm{T}_{c}=298 \mathrm{~K}, h_{c}$ is the heat transmission coefficient of the fiber surface and denotes thermal conductivity. $a$ and $b$ are the radius of the fiber core and fiber outer cladding. $\mathrm{Q}(\mathrm{z})$ is the heat power density, defined as [15]:

$$
Q(z)=\frac{\alpha(z)\left[P_{p}^{+}(z, t)+P_{p}^{-}(z, t)\right]}{\pi a^{2}}(1-S)
$$

Where $\alpha(z)=\alpha_{\alpha}(z)+\alpha_{p}, \alpha_{\alpha}(z)$ is absorption coefficient and $\mathrm{S}$ is the quantum efficiency whose theoretical value is $\lambda_{\mathrm{p}} / \lambda_{\mathrm{s}}$. However, it cannot reach the theoretical value in practical applications. In Other regions of the fiber, the value of $Q(z)$ is zero. Supposing perfect thermal connection among the inner-cladding and core, the temperature and their derivatives are continuous at the boundary $(r=a)$. The two-point boundary conditions in the above model are

$$
\begin{aligned}
& P_{p}^{+}(z=0)=P_{p 01}, P_{p}^{-}(z=L)=R_{p 2} P_{p}^{-}(z=L) \\
& P_{p}^{-}(z=L)=P_{p 02}, P_{p}^{+}(z=0)=R_{p 1} P_{p}^{-}(z=0)
\end{aligned}
$$

\begin{tabular}{|c|c|c|c|}
\hline $\boldsymbol{x}$ & $\boldsymbol{E}_{\boldsymbol{x}}\left(\mathbf{c m}^{-1} \mathbf{)}\right.$ & $\boldsymbol{v}\left(\mathbf{G H}_{\mathbf{z}}\right)$ & $\mathbf{h} \mathbf{v}(\mathbf{J})$ \\
\hline$g$ & 11,630 & 348,900 & $2.3132 \mathrm{E}^{-19}$ \\
\hline$f$ & 11,000 & 330,000 & $2.1879 \mathrm{E}^{-19}$ \\
\hline$e$ & 10,260 & 307,800 & $2.0407 \mathrm{E}^{-19}$ \\
\hline$d$ & 1490 & 44700 & $2.9636 \mathrm{E}^{-20}$ \\
\hline$c$ & 1060 & 31800 & $2.1083 \mathrm{E}^{-20}$ \\
\hline$b$ & 600 & 18000 & $2.1083 \mathrm{E}^{-20}$ \\
\hline$a$ & 0 & 0 & 0 \\
\hline
\end{tabular}

Table 1: Energy levels of $\mathrm{Yb}^{3+}$-doped fiber. 


$$
\begin{aligned}
& P_{p}^{+}(z=0)=P_{p 01}, P_{p}^{-}(z=L)=P_{p 02} \\
& P_{S}^{+}(z=0)=R_{S 1} P_{S}^{-}(z=0), P_{S}^{-}(z=L)=R_{S 2} P_{S}^{+}(z=L) \\
& P_{B}^{+}(z=0)=R_{B 1} P_{B}^{-}(z=0), P_{B}^{-}(z=L)=R_{B 2} P_{B}^{+}(z=L)
\end{aligned}
$$

\section{Simulation Results and Discussion}

The data used in calculations are $\lambda_{\mathrm{p}}=975 \mathrm{~nm}, \lambda_{\mathrm{s}}=1080 \mathrm{~nm}, \mathrm{R}_{\mathrm{ls}}=0.98$, $\mathrm{R}_{2 s}=0.04, \mathrm{~L}=5 m, \tau=0.8 m s, \sigma_{a p}=2 \times 10^{-24}{ }^{\mathrm{p}} \mathrm{m}^{2}, \sigma_{e p}=2 \times 10^{-24} \mathrm{~m}^{2}, \Gamma_{P}=0.0012$,
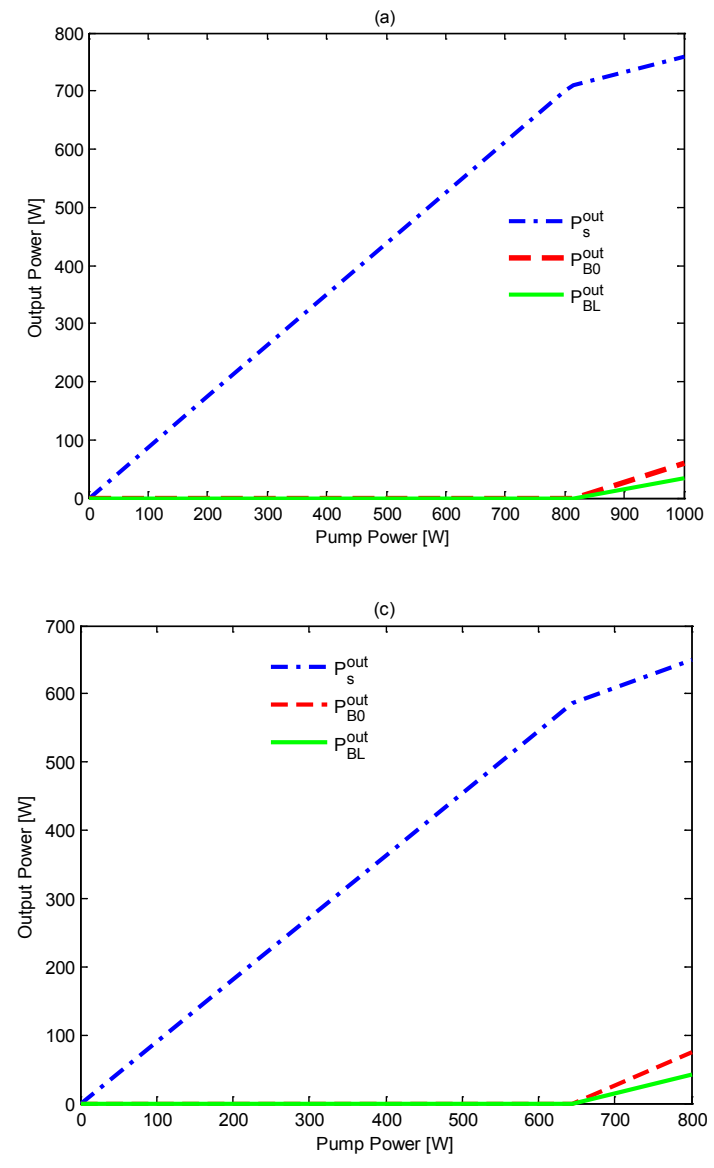

$\sigma_{a s}=3.1 \times 10^{-27} \mathrm{~m}^{2}, \quad \sigma_{e s}=4.2 \times 10^{-25} \mathrm{~m}^{2}, \quad \Gamma_{s}=0.8, \quad N=1.6 \times 10^{26}$, $\alpha_{s}=5 \times 10^{-3} \mathrm{~m}^{-1}, \alpha_{p}=3.1 \times 10^{-3} \mathrm{~m}^{-1}$ fiber core radius $D=10 \mu \mathrm{m}$ and $\mathrm{NA} 0.05$.

Note that bidirectional end-pumping, forward pump, forward pump with reflexion, backward pump and backward pump with reflexion are discussed. The forward pump power $\mathrm{P}_{\mathrm{p} 01}$ equal to the backward pump power $\mathrm{P}_{\mathrm{p} 02}$ in the simulation model. The laser output power $\mathrm{P}^{\text {out }}$, backward Stokes power $P_{B}^{-}(z)$ and forward Stokes power $P_{B}^{+}(z)$ as a function of pump power with temperature factor at different pump schemes are depicted in Figure 3a-3e. The SBS occurs once the pump
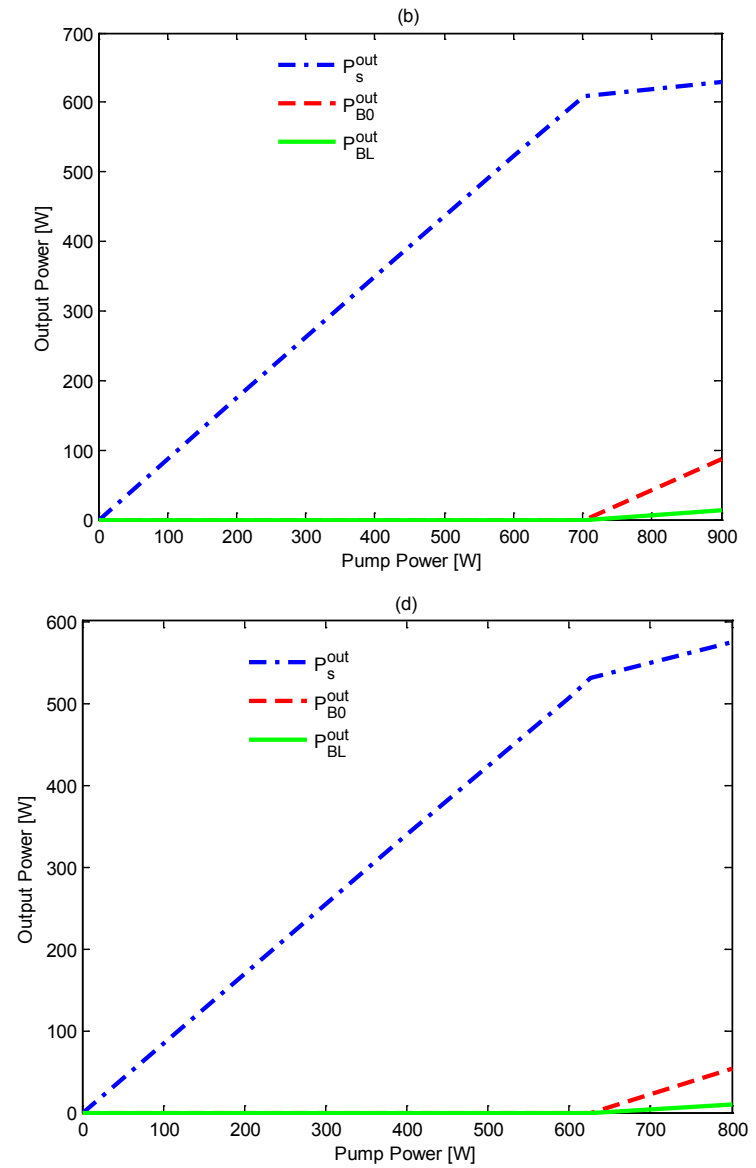

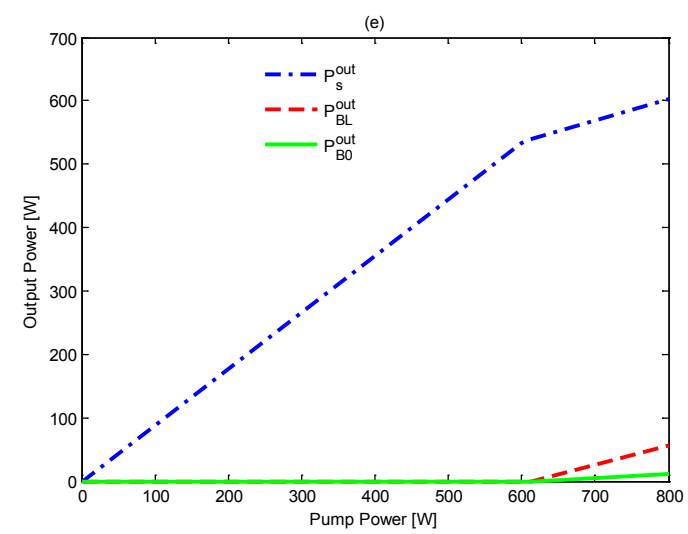

Figure 3: Output laser and Stokes power for different pump schemes: (a) Bidirectional pump scheme; (b) Forward pump scheme with $R_{\mathrm{p} 2}=0$.; (c) Forward pump scheme with $R_{p 2}=0.98$; (d) Backward pump scheme with $R_{p 1}=0$; (e) Backward pump scheme with $R_{p 1}=0.98$. 


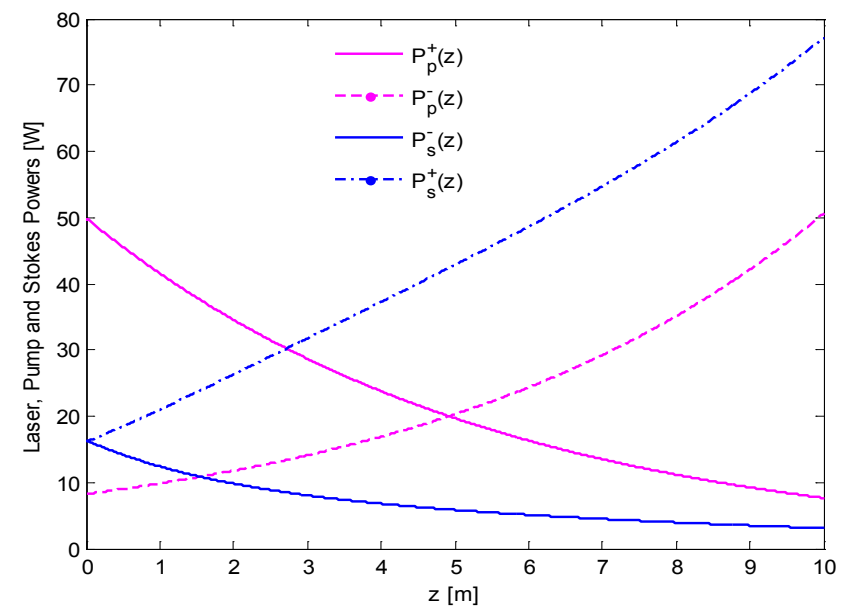

Figure 4: Laser, pump and Stokes powers propagating along axial position, with fiber length $10 \mathrm{~m}$ and a pump power is $100 \mathrm{~W}$.

power reaches the Brillouin threshold power. When the pump power exceeds the Brillouin threshold power, the laser conversion efficiency starts to drop due to the presence of the forward and backward Stokes waves. The Brillouin threshold power and the corresponding laser output powers increases as the pump power increases. The Brillouin threshold powers considering temperature factor are respectively $825 \mathrm{~W}$ for the bidirectional pump scheme in Figure 3a, $702 \mathrm{~W}$ for the forward pump scheme without reflection in Figure 3b, $644 \mathrm{~W}$ for the forward pump scheme with reflection in Figure 3c, $627 \mathrm{~W}$ for the backward pump scheme without reflection in Figure 3d, and $612 \mathrm{~W}$ for the backward pump scheme with reflection in Figure 3e. In addition, the lasers outputs are correspondingly $771.63 \mathrm{~W}, 608.04 \mathrm{~W}, 536.02 \mathrm{~W}$, $530.07 \mathrm{~W}$ and $540.31 \mathrm{~W}$.

The pump power $P_{p}^{ \pm}(z)$, signal power $P_{s}^{ \pm}(z)$ and Stokes power $P_{B}^{ \pm}(z)$ propagating along fiber axial position with bidirectional pump scheme and with pump power equal to $100 \mathrm{~W}$, are shown in Figure 4. The stokes power not appear, for the pump power of $100 \mathrm{~W}$, because this value of the pump power not exceeds the threshold stokes power.

\section{Conclusion}

The Stimulated Brillouin Scattering (SBS) of linear cavity highpower $\mathrm{Yb}^{3+}$-doped photonic crystal fiber lasers has been studied Numerically. By solving the rate equations with SBS, we have investigated the effects of pump schemes mode.

Numerical results show that the SBS threshold power can be improved significantly by the bidirectional pump scheme and the pump with reflectivety minimize the SBS threshold slightly in the both pump schems forward and backward pump scheme with reflexion, compared with the both forward and backward pumps schemes respectivly.

\section{References}

1. DeLoach LD, Payne SA, Chase LL, Smith LK, Kway WL, et al. (1993) Evaluation of absorption and emission properties of $\mathrm{Yb}^{3+}$-doped crystals for laser applications. IEEE J Quantum Electron 29: 1179-1191
2. Zenteno L (1993) High-power double-clad fiber lasers. J Light wave Tech 11: $1435-1446$.

3. Limpert J, Liem A, Zellmer H (2003) 500W continuous wave fiber laser with excellent beam quality. Electron Lett 39: 645-647.

4. Jeong Y, Sahu JK, Baek S, Alegria C, Soh DBS, et al. (2004) Claddingpumped ytterbium-doped large-core fiber laser with $610 \mathrm{~W}$ of output power. Opt Commun 234: 315-319.

5. Jeong Y, Sahu J, Payne D, Nilsson J (2004) Ytterbium-doped large-core fiber laser with $1.36 \mathrm{~kW}$ continuous-wave output power. Opt Express 12: 6088-6092.

6. Salhi M, Hideur A, Chartier T, Brunel M, Martel G, et al. (2002) Evidence of Brillouin scattering in an ytterbium-doped double-clad fiber laser. opt Lett 27 : 1294-1296.

7. Hideur A, Chartier T, Brunel M, Louis, S, Özkul C, et al. (2001) Generationof high energy femtosecond pulses from a side-pumped $\mathrm{Yb}$ - doped double-clad fiber laser. Appl Phys Lett 79: 3389-3391.

8. Karpov VI, Dianov EM, Paramonov VM, Medvedkov OI, Bubnov MM, et al (1999) Laser-diode-pumped phosphosilicate-fiber Raman laser with an output power of 1W $1.48 \mathrm{~mm}$. Opt Lett 24: 887-889.

9. Chen ZJ, Grudinin AB, Porta J, Minelly JD (1998) Enhanced Q switching in double-clad fiber lasers. Opt Lett 23: 454-456.

10. Hekmat M, Dashhtabi M, Manavi S, Hassanpour E, Massudi R (2013) Study of the stimulated Brillouin scattering power threshold in high power double-clad fiber lasers. Laser Phys 23: 025104.

11. Yang H, Duan K, Zhao B, Zhang E (2013) Theoretical study of stimulated Brillouin scattering in high-power dual-clad fiber lasers. Optik 124: 1049-1052.

12. liu G, Liu D (2009) Numerical analysis of stimulated Brillouin scattering in highpower double-cald fiber lasers. Optik 120: 24-28.

13. Hu X, Ning T, Chen Q, Li J (2015) Stimulated Brillouin scattering in Yb3+ -doped dual-clad fibe $r$ lasers based on the temperature-dependent model. optic 126: 50-55.

14. Hideur A, Chartier T, Ozkul C, Sanchez F (2000) Dynamics and stabilization of a high power side-pumped $\mathrm{Yb}$ doped double-clad fiber laser. Opt Commun 186: 311-317.

15. Shimizu K, Horiguchi T, Koyamada Y (1992) Coherent light-wave amplification and stimulated Brillouins catteringin an erbium-doped fiber amplifier. IEEE Photon Tech Lett 4: 564-567.

16. Shao H, Duan K, Zhu Y, Yan H, Yang H (2013) Numerical analysisof Ytterbium doped double-clad fiber lasers based on the temperature-dependent rate equation. Optik 124: 4336-4340.

17. Xiao L, Yan P, Gong M, Wei W, Ou P (2004) An approximate analytic solution of strongly pumped $\mathrm{Yb}$-doped double-clad fiber lasers without neglecting the scattering loss. Opt Commun 230: 401-410.

18. Marcuse D (1978) Gaussian approximation of the fundamental modes of graded-index fibers. J Opt Soc Am B 68: 103-109.

19. Agrawal GP (2010) Fiber-optic Communication Systems, John Wiley \& Sons, NewYork.

20. Husein AHM, El-Astal AH, EL-Nahal FI (2011) The gain and noise figure of $\mathrm{Yb}-\mathrm{Er}$-codoped fiber amplifiers based on the temperature-dependent model. Opt. Mater 33 543-548

21. Peng $X$, Dong $L$ (2008) Temperature dependence of ytterbium-doped fiber amplifiers. J Opt Soc Am B 25: 126-130.

22. Brilliant NA, Lagonik $K$ (2001) Thermal effects in a dual-clad ytterbium fiber laser. Opt Lett 26 1669-1671.

23. Abouricha M, Boulezhar A, Habiballah NC (2013) The Comparative Study of the Temperature Distribution of Fiber Laser with Different Pump Schemes. Open J Metal 3: 64-71 